Basic Neuroscience

\title{
Using the readiness potential of button-press and verbal response within spoken language processing
}

\author{
Stefanie Jansen ${ }^{\mathrm{a}, \mathrm{c}, *}$, Hendrik Wesselmeier ${ }^{\mathrm{a}, \mathrm{c}}$, Jan P. de Ruiter ${ }^{\mathrm{b}, \mathrm{c}}$, Horst M. Mueller ${ }^{\mathrm{a}, \mathrm{c}}$ \\ a Experimental Neurolinguistics Group, Faculty of Linguistics and Literary Studies, Bielefeld University, Bielefeld, Germany \\ b Psycholinguistics Group, Faculty of Linguistics and Literary Studies, Bielefeld University, Bielefeld, Germany \\ ' Collaborative Research Centre "Alignment in Communication" (SFB 673), Bielefeld University, Bielefeld, Germany
}

\section{H I G H L I G H T S}

- Detection of readiness potential onset represents a preconscious measure for end-of-turn anticipation in a language dialogue.

- Even if it is a language task it can be applied equally well to both verbal and finger movement responses.

- In contrast to behavioural reaction time tasks the EEG-measurement produces more reliable data for the anticipation performance in end-of-turndetection.

\section{A R T I C L E I N F O}

\section{Article history:}

Received 9 December 2013

Received in revised form 23 April 2014

Accepted 24 April 2014

\section{Keywords:}

EEG

Turn-taking

Event-related potential

Button-press and verbal responses

Language processing

\begin{abstract}
A B S T R A C T
Background: Even though research in turn-taking in spoken dialogues is now abundant, a typical EEGsignature associated with the anticipation of turn-ends has not yet been identified until now.

New method: The purpose of this study was to examine if readiness potentials (RP) can be used to study the anticipation of turn-ends by using it in a motoric finger movement and articulatory movement task. The goal was to determine the preconscious onset of turn-end anticipation in early, preconscious turn-end anticipation processes by the simultaneous registration of EEG measures (RP) and behavioural measures (anticipation timing accuracy, ATA). For our behavioural measures, we used both button-press and verbal response ("yes"). In the experiment, 30 subjects were asked to listen to auditorily presented utterances and press a button or utter a brief verbal response when they expected the end of the turn. During the task, a 32-channel-EEG signal was recorded.

Results: The results showed that the RPs during verbal- and button-press-responses developed similarly and had an almost identical time course: the RP signals started to develop $1170 \mathrm{vs.} 1190 \mathrm{~ms}$ before the behavioural responses.

Comparison with existing methods: Until now, turn-end anticipation is usually studied using behavioural methods, for instance by measuring the anticipation timing accuracy, which is a measurement that reflects conscious behavioural processes and is insensitive to preconscious anticipation processes. Conclusion: The similar time course of the recorded RP signals for both verbal- and button-press responses provide evidence for the validity of using RPs as an online marker for response preparation in turn-taking and spoken dialogue research.
\end{abstract}

(C) 2014 Elsevier B.V. All rights reserved.

\section{Introduction}

A number of different neural substrates involved in language processing account for the high efficiency needed in human

\footnotetext{
* Corresponding author at: Experimental Neurolinguistics Group (SFB 673), Bielefeld University, P.O. Box 100131, 33502 Bielefeld, Germany. Tel.: +49 05211063182; fax: +490521106155307

E-mail address: stefanie.jansen@uni-bielefeld.de (S. Jansen).
}

communication processes, for example in sound discrimination and perception, semantic-pragmatic analysis or meaning constitution (Friederici, 2004; Indefrey and Levelt, 2004; Müller, 2006). For example, the transfer of meaning in natural utterances can be detected already about $120 \mathrm{~ms}$ after articulation has started (Müller and Kutas, 1996). Considering, however, the amount of sequential information included in the acoustic signal (at the level of phonemes, syllables, words, phrases, utterances, etc.), it is obvious that the auditory system needs more processing time than that. In fact, the processing time required by the auditory system is 
almost as long or even longer than the time needed for articulation. The question why we can nevertheless communicate so quickly and efficiently is a puzzle that has been the centre of much theoretical and experimental research (see e.g., Levinson, 2000; Ford and Thompson, 1996). This high efficiency of communication and, especially, the time course of the different parallel and sequential stages of language processing has been a main topic of research over the last couple of years. Results show that cognitive parsing of the perceived utterances follow the acoustic speech signal within a few tenths of a second (see e.g., Friederici, 2004; Indefrey and Levelt, 2004; Müller, 2006). To achieve such a short timeframe in a spoken dialogue, the prediction and anticipation of one interlocutor's turn-end is required (De Ruiter et al., 2006).

\subsection{Realistic time frames for turn-end-detection}

Since Sacks et al. (1974) developed their turn-taking model of conversation, several studies have examined key aspects of conversation (De Ruiter et al. 2006), and found that listeners must perform several tasks simultaneously during conversation. Besides other things, a listener must comprehend the speaker's turn, while at formulating a reply and pre-planning the onset of its articulation. The latter process requires quite precise timing, to minimize gaps and overlaps (Stivers et al., 2009; Magyari and De Ruiter, 2008, 2012). These studies found, among other things, that gaps and overlaps are usually shorter than $250 \mathrm{~ms}$. As it is not possible to sequentially listen to a turn, comprehend it, prepare a response, and initiate this response within such a short time window (especially in case of overlaps), the authors assume that interlocutors use incremental and possibly overlapping processes to be able to time an appropriate response sufficiently accurate. Further evidence for this assumption comes from the famous shadowing task by Marslen-Wilson $(1973,1985)$, in which people were able to repeat another speaker's sentences with a time delay of only $250 \mathrm{~ms}$. Finally Pulvermüller resumes that early indexes of lexical, syntactic and semantic processes have been found after 100-250 ms in written and spoken language processing which reflects almost parallel processes (Pulvermüller, 2005; Pulvermüller et al., 2009). Both behavioural studies (Marslen-Wilson, 1985) as well as the results of the underlying functional neuroanatomical studies are therefore roughly comparable to the observed behavioural inter-turn delays of about $120-250 \mathrm{~ms}$. However, the frequently occurring precise or even premature initiation of subsequent turns (e.g., De Ruiter et al., 2006) can only be explained by anticipation.

The time course of language processing is often estimated on the basis of the observation of behavioural output. As neurocognitive evidence reveals, though, language processes are faster and start much earlier than behavioural data might suggest. Müller and Kutas (1996), for example, showed that the initial $100-120$ ms of words already provide enough information in order to decide whether a sound is the beginning of a noun or a name. In another study McGregor et al. (2012) investigated the crucial point of word recognition in spoken words versus pseudowords. They wanted to find out about the point in time when the acoustic information allows word recognition. Results showed that this crucial point occurs 50-80 ms after presentation (McGregor et al., 2012). In a word reading task that compared different semantic word classes with similar physical appearance by using textual characters (Chinese), electrical brain activation differed significantly for each semantic word class. For these visually presented Chinese characters, the earliest neural signature appeared $80 \mathrm{~ms}$ after stimulus onset (Skrandies et al., 2004). Dell'Acqua et al. (2010) achieved similar results when they studied the activation time of semantic and phonological representation. The obtained ERP results showed a primary component and a later, distinct component. This has lead to the assumption of a bimodal distribution of scalp activity for the semantic effects. The primary component was characterized by a fast onset with a sharp increase during the first $50 \mathrm{~ms}$ past stimulus onset and a decrease after $200 \mathrm{~ms}$ (Dell'Acqua et al., 2010). Although this working group usually focuses on a variety of components, their results correspond with those from the previously mentioned studies regarding the timing of processing. Irrespective of the specific nature of the language processing task, all examples show that processing starts between 50 and $200 \mathrm{~ms}$ post stimulus onset. Therefore, it is reasonable to suspect that turn-end anticipation follows a similar time course.

In contrast to the aforementioned findings about the time course of language comprehension, the results of word production experiments reveal that much more time is needed for processing. For instance, Indefrey and Levelt (2004) found that it takes the production system $600-1200 \mathrm{~ms}$ to get from an intention to speak to the actual articulation of words. During natural language processing, especially in interactive situations like dialogue, there are a number of simultaneous processes required, for example comprehension and production processes. Almost all of these processes are very fast, and overlap in time, which is presumably possible because of the massive, fine-grained parallelism in the neural computations in the brain.

The subject of our investigation is the time course of the preconscious processing that takes place before the behavioural response. It is important to define the latter carefully, because even though we can measure the behavioural response well, in anticipation processes the exact start of the associated "stimulus" is unknown. This means that the behavioural responses we are interested in do not correspond with reaction times as found in psycholinguistic tasks such as lexical decision or picture naming. Therefore, our primary behavioural dependent measure is the Anticipation Timing Accuracy (ATA), which is defined as the point in time at which the response is recorded, minus the point in time that the stimulus (the presented turn) actually ends. So if a participant's timing is perfect, the ATA is 0 , if the participant responds too early (i.e., before the end of the turn), it is negative, and if they respond too late (after the end of the turn), it is positive. This dependent measure is the same as what De Ruiter et al. (2006) termed BIAS.

\subsection{Previous EEG studies}

An EEG-study by Magyari et al. (2011) used a spectral analytic technique for analysing EEG-recordings in a behavioural task of anticipation processes in turn-taking. They presented conversational turns with an average duration of $2.9 \mathrm{~s}$ that varied in the predictability of their ending while recording EEG data. ATA was evaluated by a button-press at the turn-end and were indeed found to be more precise for the turns with more predictable endings. Furthermore, they identified a beta power decrease in the predictable condition $1700 \mathrm{~ms}$ before the actual button-press as well as a beta power increase during the same time interval. These results support the assumption that the accuracy of turn-end anticipation is related to the accuracy of predictions about upcoming words. In another EEG-study (Galgano and Froud, 2008) event-related potentials in preparation for voice onset as well as exhalation were analyzed in a stimulus-induced voluntary movement task. The results showed a slow, increasingly negative cortical potential in the time window preceding the onset of phonation. These results reveal the benefits of RPs as a slow negative-going cortical potential correlated with the preparation of voluntary movements, especially with voicerelated initiation (Galgano and Froud, 2008). Since the discovery of the RP ("Bereitschaftspotential") in 1965 (Kornhuber and Deecke, 1965) several studies have provided evidence for a RP preceding speech-related volitional motor acts (e.g., Galgano and Froud, 2008). Therefore, the RP is defined as an ERP-component, which 
is related to selective response activation processes. Many studies provide insights into temporal aspects in language processing by ERP analysis (e.g., Swaab et al., 2012) or spectralanalytic techniques (e.g., Schack et al., 2003) but only a few are using a RP in this context (e.g. Brunia et al., 2012; McArdle et al., 2009). However, even if it is possible to investigate RPs in an auditory experimental setting it is still unclear if it could be evaluated in a natural language production task including articulator movement. Consequently, this study examines turn anticipation using the RPs of the given ATA-responses in an auditory task as correlative for verbal or motor responses. The RPs serve as our dependent measure, in addition to the recorded behavioural data.

These considerations permit us to specify our main research questions: (A) Can we use RPs to detect the early onset of preconscious anticipation processes in a language comprehension task? (B) If so, does this work at least equally well for button-press and verbal (ATA) responses?

\section{Methods}

\subsection{Participants}

30 students (17 women, 13 men) of Bielefeld University participated in the experiment. They were native German speakers between 19 and 35 years of age ( $24.5 \pm 3.5$ years). The subjects were right-handed with a lateralization quotient of 93.5 according to The Edinburgh Handedness Inventory (Oldfield, 1971). We conducted a short face-to-face interview to assess the participants' hearing and visual faculty. Participants who used antipsychotic medication (antipsychotic drugs, any psychotropic medication or beta blocker) were excluded from the study. The participants were paid for their participation.

\subsection{Stimuli}

All experimental materials (88 stimuli) were spoken by a professional female speaker with natural intonation and recorded in a sound studio. The sentences were acoustically presented via loudspeaker. The mean stimulus intensity ranged between 55 and $60 \mathrm{~dB}$ and therefore corresponded with that of a normal face to face conversation.

The experimental material contained 45 basic questions and 43 declarative sentences. The 88 sentences had a duration that varied between $1300 \mathrm{~ms}$ and $6643 \mathrm{~ms}$ ( $\varnothing 4038 \mathrm{~ms}$ ). The number of words in the sentences ranged from 3 to 22 ( $\varnothing 12.11)$. The subjects were asked to give a short verbal answer ("Ja" or "Nein") in the first experimental block. In a second block, they were asked to signal their expectation of the turn-end by pressing a button (motor-response) at the end of each sentence as quickly as possible. To contrast the verbal responses with the button-press responses consistently, we excluded responses that took more than $250 \mathrm{~ms}$. All stimuli including the fixation cross were presented by a customized, Linux-based presentation programme (Sculptor).

\subsection{Procedure}

Participants were comfortably seated in front of a computer screen. A USB-button-box with an internal clock was positioned beneath their right hand. They were instructed to sit still while looking at the fixation cross on the screen in front of them. The sentences started 1000-2500 ms (randomized) after the fixation cross appeared on the screen. The fixation cross disappeared $1000 \mathrm{~ms}$ after the sentence ended.

We investigated the temporal aspects in turn anticipation by comparing finger movement (button-press) of the right index finger and articulator-movement (verbal response). The voice key was entered manually. We were able to specify the exact beginning of the articulation according to the recorded microphone track.

A total of 88 auditorily presented stimuli were used including 25 questions and 20 declarative sentences demanding a verbal response and 43 declarative sentences which required buttonpress responses by using a bounce-free pushbutton.

\subsection{EEG recordings}

The EEG recordings were conducted in a soundproof and electromagnetically shielded booth. EEGs were recorded from 32 active-scalp electrodes embedded in a cap (ActiCap, Brain Products). Two electrodes were fixed on the left and right canthi, one was fixed vertically (supra-orbitally) below the right eye, and 2 affixed to the mastoids bilaterally. Signals were sampled at $1000 \mathrm{~Hz}$ and amplified with a $50 \mathrm{~Hz}$ notch filter and a bandpass of $0.16-80 \mathrm{~Hz}$ (QuickAmp, Brain Products). Impedance was kept below $5 \mathrm{k} \Omega$ for all channels. Motoric responses (button-press) were recorded using a USB-button-box. Verbal responses were recorded by microphone and added to the EEG recording as a separate channel.

\subsection{Data analysis}

Recorded EEG data was screened for artefacts via visual inspection using the Brain Vision Analyzer 2.0 (Brain Products). The beginning and end of each critical and control sentence were identified and marked for each of the 30 participants. The verbal responses and button-presses were also marked. For statistical analysis, a marker-table was exported such that the relevant epochs were available. For each condition, the minimum and maximum response times were recorded and the mean RT and SD were calculated (Table 1). All reaction times exceeding $250 \mathrm{~ms}$ were excluded (less than $5 \%$ in each case). All analyses and calculations were done by SPSS (version 20, IBM) under Mac OS X.

Prior to analysis, data were re-referenced to the average of the signal on all channels at each time point. Every trial was inspected semiautomatically by using the Brain Vision Analyzer 2.0 software and completed by a visual inspection for artefacts (rejected segments $\sim 15 \%$ ). As a first step of displaying the ERP data all verbal responses were averaged and displayed followed by the ERP of all motor responses. For RP detection, the average for all epochs was calculated separately for both conditions. The RPs of all 30 participants were evaluated among the grand average of all data. All EEG-analyses were done with BrainVision Analyzer 2.0. To evaluate the onsets of RPs, a combination of regression analyses and $t$-tests was done to define the RP onset as a deviation from the baseline (SPSS 20). The applied method corresponds to that of Schwarzenau et al. (1998) and defines the LRP onset as a kind of "break point" between the two intersecting straight lines that were fitted to the RP waveform. This means that one line is fitted to the baseline - equivalent to a "pre-onset" line - (by $t$-test) and the other line is fitted to the segment that rises to the peak (by linear regression). The resulting point of intersection defines the RP onset (Mordkoff and Gianaros, 2000; Schwarzenau et al., 1998).

Table 1

Descriptive statistics for all sentences ( $n$, number of involved items; ATA, anticipation timing accuracy; Min, lowest RT; Max, highest RT; SD, standard deviation).

\begin{tabular}{lllcll}
\hline Condition & $n$ & ATA $(\mathrm{ms})$ & $\operatorname{Min}(\mathrm{ms})$ & $\operatorname{Max}(\mathrm{ms})$ & SD \\
\hline 1. Verbal response & 450 & 85.7 & -857.0 & 250.0 & 141.7 \\
2. Button-press response & 653 & 50.8 & -1179.0 & 250.0 & 181.9 \\
\hline
\end{tabular}




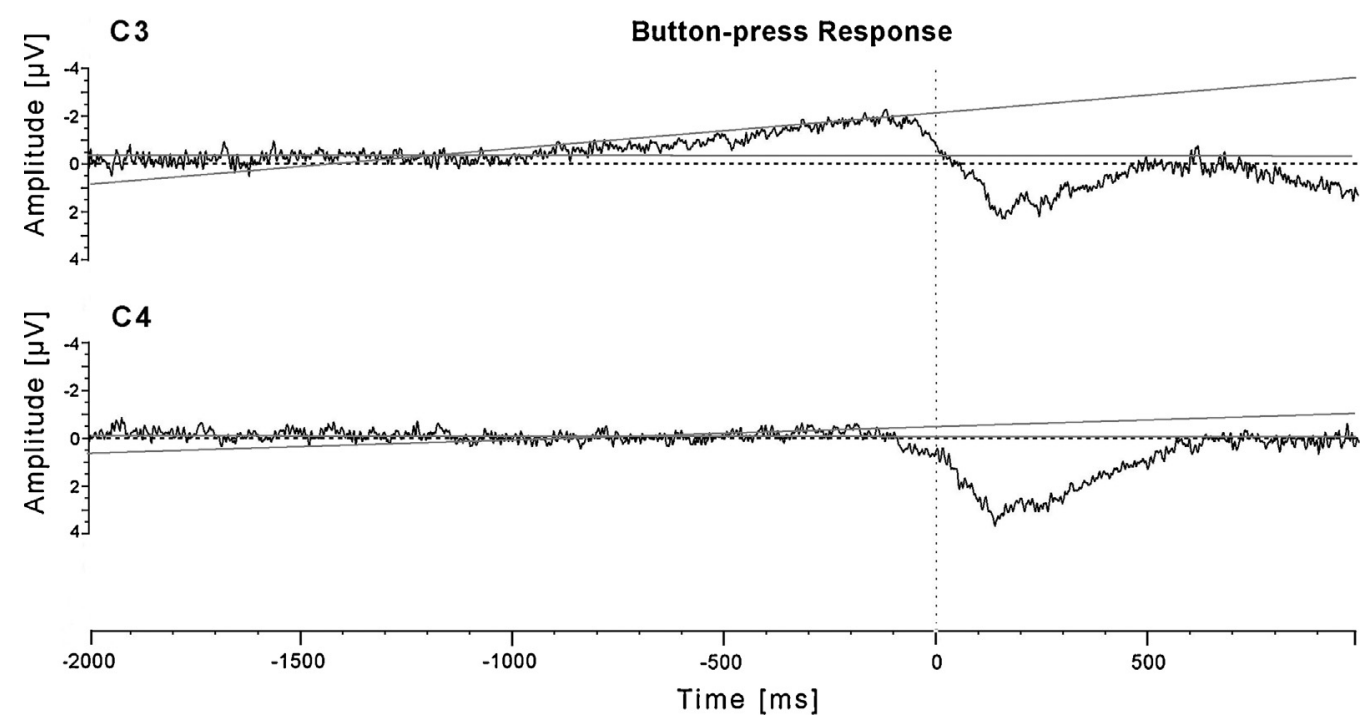

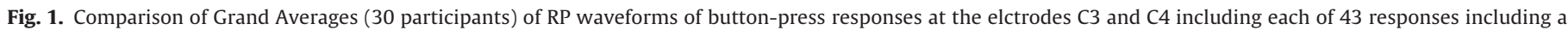
regression-based RP onset detection. The RP of the left hemisphere C3 illustrates that the right index finger was used. The RP has its estimated onset at about -1100 ms.

\section{Results}

The results of the descriptive statistical analysis are summarized in Table 1 for both verbal responses and button-press responses. For a total of 450 verbal responses and 653 buttonpress responses we calculated the mean response duration (verbal responses: 85.7; button-press responses: $50.8 \mathrm{~ms}$ ), minimal and maximal response time (verbal responses: -857.0 to $250.0 \mathrm{~ms}$; button-press responses: -1179.0 to $250.0 \mathrm{~ms}$ ), and SD. The calculations revealed a significant $(p<0.01)$ difference between the anticipation timing accuracy of the two conditions. Subjects reacted much faster in the button-press condition compared to the verbal responses.

Further calculation refers to the EEG-data. We evaluated the RPs of all verbal- and button-press responses at different electrodes. For the button-press condition we focused on the electrode positions C3 and C4 as known representatives for motor action or approximately the hand representation areas (see e.g. Pfurtscheller et al., 2006).

\subsection{Button-press condition}

Fig. 1 shows the RPs of all button-press responses from electrodes C3 and C4. For both electrodes the RP onset was determined via a baseline-deviation by $t$-test at $-1100 \mathrm{~ms}$.

\subsection{Verbal response condition}

For evaluation of the verbal responses, we also focused on the $\mathrm{Cz}$-electrode in order to allow for a direct comparison. Fig. 2 gives an overview of verbal- and button-press responses at $\mathrm{Cz}$.

In the next step the button-press responses were contrasted with the verbal responses. After determining the RP onset by a baseline regression ( $t$-test) (verbal: $-1190 \mathrm{~ms}$, button-press: $-1170 \mathrm{~ms}$ ), a regression analysis was done as well. The results exhibit the correlation between the amplitude of the two different response forms over time and illustrate the increase of the RPs (Fig. 2).

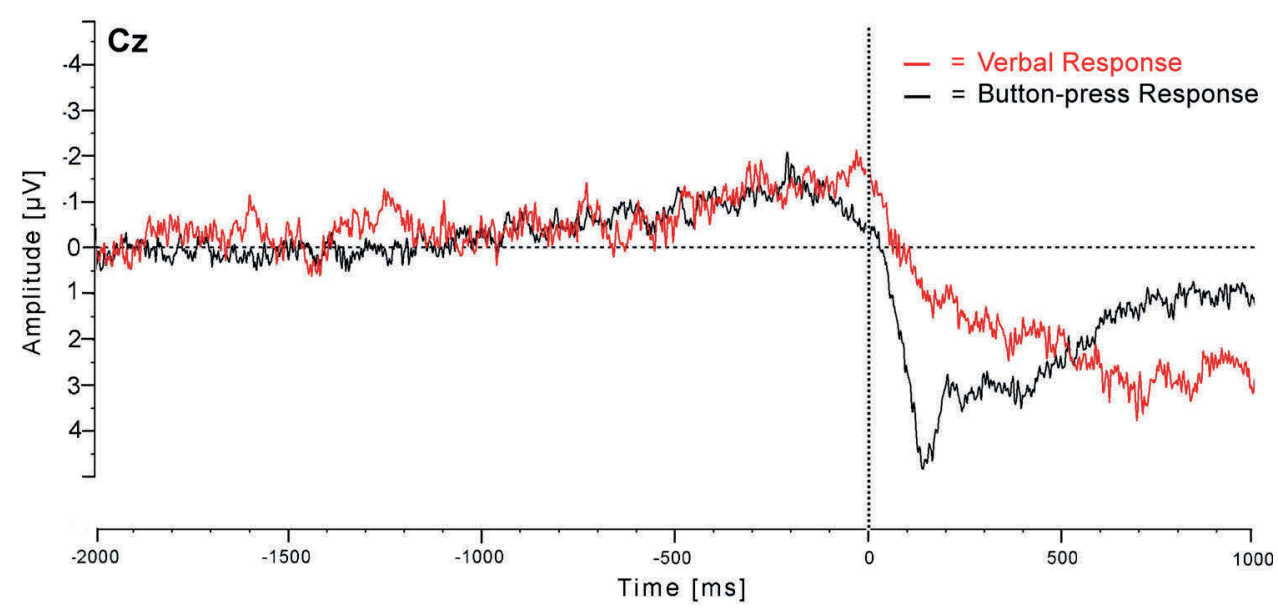

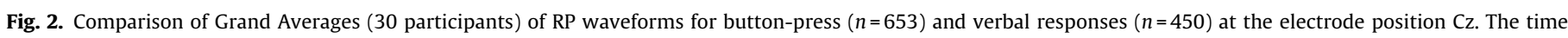
course of both RPs are quite similar. 

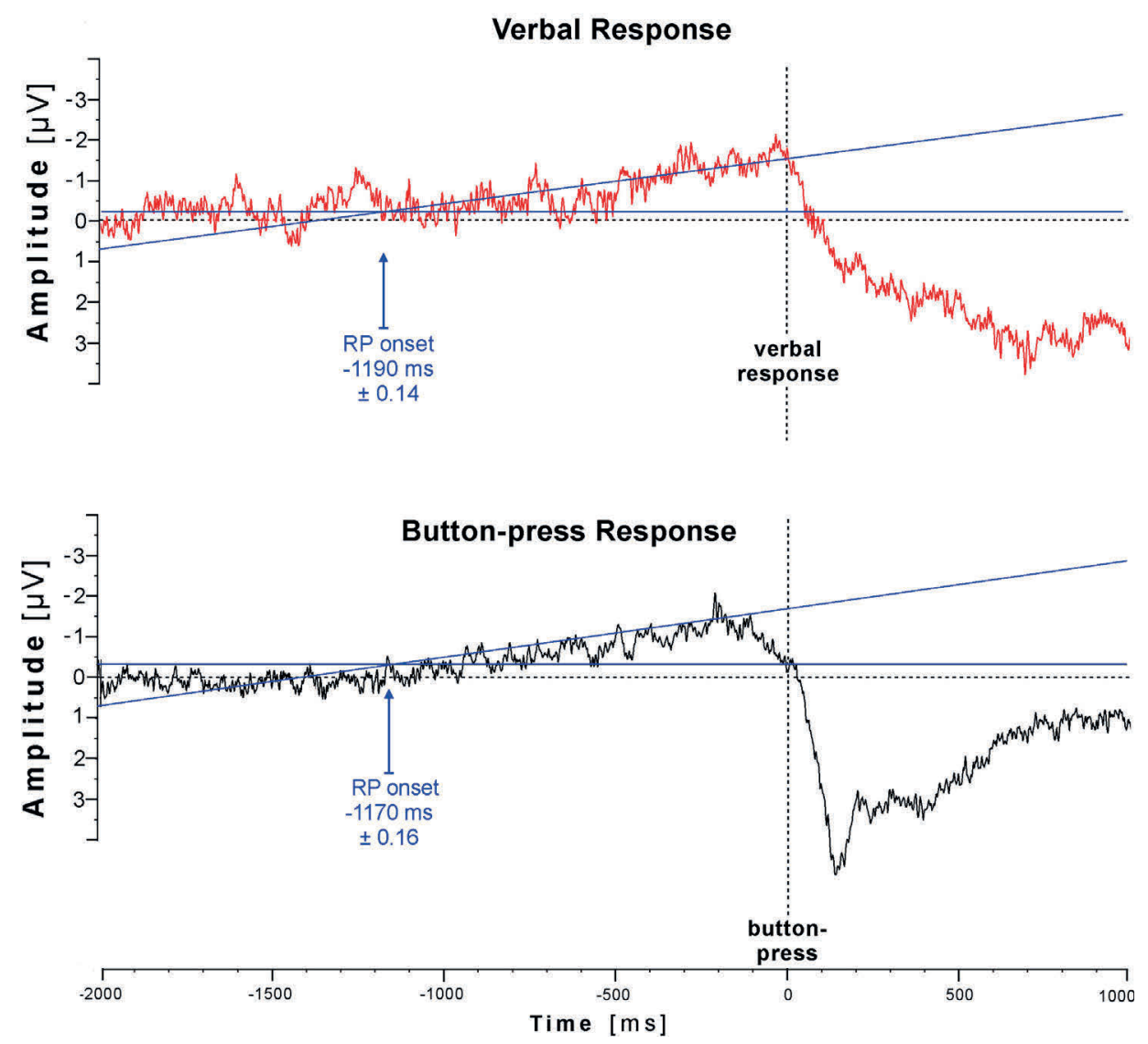

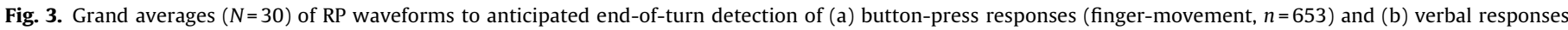
(articulatory movement “Ja" and "Nein", $n=450$ ) as well as the RP onsets (blue arrows) including a regression-based RP onset detection.

The verbal responses show an RP onset a little earlier $(-1190$ $\mathrm{ms})$ than the button-press responses $(-1170 \mathrm{~ms})$, which is compara-ble to the RPs at the C3 electrode. These results differ from the ATA-analysis exhibited in Table 1, which revealed more accurate responses in the motor-response-condition (Table 1). Again, results were evaluated by comparing the point at which the increase started to the corresponding point in the baseline amplitude (Fig. 2).

The depicted RPs reflect the calculated grand averages for each group. Fig. 3 shows the waveforms of the RPs for verbal- and buttonpress responses supplemented by regression lines.

The onset of RPs in the verbal condition was determined by baseline-deviation via a $t$-test. Afterwards we made use of the regression model by Mordkoff et al. (Fig. 2). Therefore we fitted straight lines close by the waveform. One line was fitted across the timepoints in the baseline and the other one at the timepoints where the RP rises to its peak (Mordkoff and Gianaros, 2000). The verbal RP response started developing at $-1190 \mathrm{~ms}$ and the button-press RP at $-1170 \mathrm{~ms}$, which on this time scale is remarkably similar and could be confirmed statistically and was indeed not significant (via a $t$-test; $p=0.818 ; T=-0.232 ; d f=$ 50.052). $(p=0.818 ; T=-0.232 ; d f=50.052)$.

\section{Discussion}

Our first and primary research question was weather the readiness potential (RP), which reflects early, preconscious processing, delivers a more reliable assessment of the timing of the neuronal processes underlying turn-anticipation than the conscious ATA measurement does.
According to the results, the RP onset was quite early in both conditions, appearing $1190 \mathrm{~ms}$ before verbal- and 1170 before button-press responses. Given that the presented turns were $4038 \mathrm{~ms}$ long on average and consisted of 12.11 words, the $1170-1190$ ms duration corresponds with roughly 3.5 words. This indicates that people preconsciously start to anticipate the end of a turn 3.5 words before its appearance, which means after they have heard 8.6 out of 12.11 words of a turn.

Our finding supports the assumption that the process of turn anticipation indeed starts much earlier than the behavioural data suggest, namely between $250 \mathrm{~ms}$ before and $250 \mathrm{~ms}$ after the turn end (De Ruiter et al., 2006; Heldner and Edlund, 2010). This early RP-onset was also confirmed in a Doppler imaging study of speech production by McArdle et al. (2009), who showed that a speechrelated RP starts at quite earlier latencies, approximately $2500 \mathrm{~ms}$ prior to the onset of speech.

Although our behavioural analysis shows a significant difference between the mean ATAs of the two conditions (verbal responses $85.7 \mathrm{~ms}$, button-press $50.8 \mathrm{~ms}$; Table 1 ), this difference is small and may well have been caused by the difference between the measure-ment method (button-press vs. voice key). In any case, both types of behavioural response show highly accurate anticipation, in line with findings from natural data (De Ruiter et al., 2006; Stivers et al., 2009; Heldner and Edlund, 2010). For a more accurate analysis, the presence of visual information should be included in further research. In contrast to ATA measurement, the RP measurement captures all preconscious articulation features and therefore the speed of verbal processing in its entity. This high processing speed could be explained by the fact that the processing time necessary 
Table 2

Descriptive statistics for the difference between verbal and button-press RPs ( $n$, number of involved items; ATA, anticipation timing accuracy; SD, standard deviation).

\begin{tabular}{llllll}
\hline Condition & $n$ & ATA $(\mathrm{ms})$ & $t$ & $d f$ & SD \\
\hline 1. Verbal response & 30 & -0.22 & -0.232 & 58 & 2.28 \\
2. Button-press response & 30 & -0.11 & -0.232 & 50.05 & 1.49 \\
\hline
\end{tabular}

for comprehension of natural sentences is much shorter than the time needed for motor articulation of the physical utterance of a sentence (e.g., Müller, 2006).

Our second question was whether the RP measure worked equally well for button-press and verbal responses. In contrast to the finger tapping behaviour, a verbal response is a completely different process that involves the initiation of multiple articulators in tight coordination, the early initiation of which may be more dif-ficult to pick up in the EEG signal. To compare these parameters, it was necessary to concentrate on the central electrode $(\mathrm{Cz})$ because the verbal task was expected to result in RPs in this area. For the button-press responses, we have also included the $\mathrm{C} 3$ and $\mathrm{C} 4$ elec-trodes. Fig. 1 shows a general comparison between these electrodes $\mathrm{C} 3$ and $\mathrm{C} 4$, approximately both motor areas. The RP onsets of both curves occur at about $-1100 \mathrm{~ms}$. The RPs are precisely detectable and therefore corroborate our hypothesis. The curves show that button-press responses are quite reliable and a suitable method for time determination. The results of the RP-analysis demonstrate for the first time that an evaluation of RPs from a finger-movement or even an articulator-movement task is a quite appropriate analysis for temporal aspects in turn-anticipation.

Taking a closer look at the RPs from Fig. 3 reveals that the main difference in RP between button-press and verbal responses occurred in the time interval between 250 and $0 \mathrm{~ms}$ before the actual end of the presented turn.

With regard to the onset of the RP component, results are converging both for verbal responses and button-presses. Keeping in mind that (a) an absolutely precise RP onset detection is not possible (Grötzinger et al., 1975; Falkenstein et al., 1994), and (b) the average duration of the presented turns was $4038.8 \mathrm{~ms}$, the small difference between the verbal and button-press RP (1170 vs. $1190 \mathrm{~ms}$ ) is negligible (Table 2). This leads us to conclude that the $\mathrm{RP}$ can be used for both of these behavioural measures.

\section{Conclusions}

The results of our study provide strong support for the use of RP components to complement the use of behavioural (ATA) recordings. While the behavioural data shows the overt, conscious, observable, and ecologically relevant behaviour of dialogue participants, the RP can show us when preconscious anticipation processes are initiated. The combined findings that (a) RP components can be used to tap early anticipation processes in turn-taking and (b) this works equally well for button-press as well as verbal responses, paves the way for a new approach to studying turntaking with neurocognitive methods. The most exciting application of the methodology outlined here is to study early preconscious anticipation of turn-initiation in free-form natural dialogues, with-out any need for the participants pressing buttons.

\section{Acknowledgment}

This study was supported by the German Research Foundation (DFG) within the Collaborative Research Centre "Alignment in Communication" (SFB 673).

\section{References}

Brunia CHM, Boxtel GJM, Böcker KBE. Negative slow waves as indices of anticipation: the Bereitschaftspotential, the contingent negative variation, and the stimulus-preceding negativity. In: Luck SJ, Kappenmann ES, editors. The Oxford handbook of event-related potential components. Oxford: Oxford University Press; 2012. p. 189-207.

De Ruiter JP, Mitterer H, Enfield NJ. Projecting the end of a speaker's turn: a cognitive cornerstone of conversation. Language 2006;82:515-35.

Dell'Acqua R, Sessa P, Peressotti F, Mulatti C, Navarrete E, Grainger J. ERP evidence for ultra-fast semantic processing in the picture-word interference paradigm. Front Psychol 2010;170:1-10.

Falkenstein M, Hohnsbein J, Hoormann J. Effects of choice complexity on different subcomponents of the late positive complex of the event-related potential. Electroencephalogr Clin Neurophysiol 1994;92:148-60.

Ford CE, Thompson SA. Interactional units in conversation: syntactic, intonational, and pragmatic resources for the management of turns. In: Schegloff EA, Thompson SA, editors. Interaction and grammar. Cambridge: Cambridge University Press; 1996. p. 135-84.

Friederici AD. Lateralisation of auditory language functions: a dynamic dual pathway model. Brain Lang 2004;89:267-76.

Galgano J, Froud K. Evidence of the voice-related cortical potential: an electroencephalographic study. Neuroimage 2008;41:1313-23.

Grötzinger B, Kornhuber HH, Kriebel J. Methodological problems in the investigation of cerebral potentials preceding speech: determining the onset and suppressing artefacts caused by speech. Neuropsychologia 1975;13:263-70.

Heldner M, Edlund J. Pauses, gaps and overlaps in conversations. J Phon 2010:38:555-68.

Indefrey P, Levelt WJM. The spatial and temporal signatures of word production components. Cognition 2004;92:101-44.

Kornhuber HH, Deecke L. Hirnpotentialänderungen bei Willkürbewegungen und passiven Bewegungen des Menschen: Bereitschaftspotential und reafferente Potentiale. Eur J Physiol 1965;281:1-17.

Levinson S. Presumptive meanings: the theory of generalized conversational implicature. Cambridge: MIT Press; 2000.

Magyari L, De Ruiter JP. Timing in conversation: the anticipation of turn endings. In: Ginzburg J, Healey P, Sato Y, editors. Proceedings of the 12th workshop on the semantics and pragmatics dialogue. London: King's college; 2008. p. 139-46.

Magyari L, De Ruiter JP. Prediction of turn-ends based on anticipation of upcoming words. Front Psychol 2012;3:376.

Magyari L, Bastiaansen MCM, De Ruiter JP, Levinson SC. Neuronal correlates of anticipation related to turn-taking in conversations. In: Poster presented at conference architectures and mechanisms for language processing (AMLaP); 2011.

Marslen-Wilson W. Linguistic structure and speech shadowing at very short latencies. Nature 1973;244:522-3.

Marslen-Wilson WD. Speech shadowing and speech comprehension. Speech Commun 1985:4:55-73.

McArdle JJ, Mari Z, Pursley RH, Schulz GM, Braun AR. Electrophysiological evidence of functional integration between the language and motor system in the brain: study of the speech Bereitschaftspotential. Clin Neurophysiol 2009;120:275-84.

McGregor LJ, Pulvermüller F, van Casteren M, Shtyrov Y. Ultra-rapid access to words in the brain. Nat Commun 2012;3:711.

Mordkoff JT, Gianaros PJ. Detecting the onset of the lateralized readiness potential: a comparison of available methods and procedures. Psychophysiology 2000;37:347-60.

Müller HM. Neurobiological aspects of meaning constitution during language processing. In: Rickheit G, Wachsmuth I, editors. Trends in linguistics: situated communication. Berlin: Mouton de Gruyter; 2006. p. 243-64.

Müller HM, Kutas M. What's in a name? Electrophysiological differences between spoken nouns, proper names, and one's own name. NeuroReport 1996:8:221-5.

Oldfield RC. The assessment and analysis of handedness: the Edinburgh inventory. Neuropsychologia 1971;9:97-113.

Pfurtscheller G, Brunner C, Schlögl A, Lopes da Silva FH. Mu rhythm (de)synchronization and EEG single-trial classification of different motor imagery tasks. NeuroImage 2006;31:153-9.

Pulvermüller F. Brain mechanisms linking language and action. Nat Rev Neurosc 2005;6:576-82.

Pulvermüller F, Shtyrov Y, Hauk O. Understanding in an instant: neurophysiological evidence for mechanistic language circuits in the brain. Brain Lang 2009;110:81-94.

Sacks H, Schegloff EA, Jefferson G. A simplest systematics for the organization of turn-taking for conversation. Language 1974;50:696-735.

Schack B, Weiss S, Rappelsberger P. Cerebral information transfer during word processing: where and when does it occur and how fast is it? Hum Brain Map 2003:19:18-36.

Schwarzenau P, Falkenstein M, Hoormann J, Hohnsbein J. A new method for the estimation of the onset of the laterized readiness potential (RP). Behav Res Methods Instrum Comput 1998;30:110-7.

Skrandies W, Chiu MJ, Lin YR. The processing of semantic meaning in Chinese words and evoked brain topography. Brain Topogr 2004;16:255-9.

Stivers T, Enfield NJ, Brown P, Englert C, Hayashi M, Heinemann T, et al. Universals and cultural variation in turn-taking in conversation. Proc Natl Acad Sci USA 2009;106:10587-92.

Swaab TY, Ledoux K, Camblin CC, Boudewyn MA. Language-related ERP components. In: Luck SJ, Kappenmann ES, editors. The Oxford handbook of event-related potential components. Oxford: Oxford University Press; 2012. p. 397-439. 